\title{
Infection on a Gynecologic Oncology Service
}

\author{
S. Gene McNeeley, Jr., M.D., ${ }^{1}$ Michael P. Hopkins, M.D., Barbara Ehlerova, M.D., \\ AND JAMES ROBERTS, M.D. \\ Department of Obstetrics and Gynecology, University of Michigan, 1500 East Medical Center Drive, Ann Arbor, Michigan 48109-0718
}

Received April 25, 1989

\begin{abstract}
Little information is available pertaining to infectious morbidity in women with genital malignancy. To define the magnitude of this problem, all patients admitted to the gynecologic oncology services at the University of Michigan Medical Center between January 1, 1986, and December 31, 1986, were followed prospectively for the development of infectious morbidity. One hundred nine bacteriologically confirmed infections occurred in 297 patients during 510 admissions. An additional 31 postoperative patients received empiric therapy for presumed infection. Urinary tract (54) and wound (22) infections were the most commonly confirmed infections. The pathogens isolated from oncology patients were significantly different in frequency of isolation and antibiotic sensitivity when compared with pathogens isolated from women developing infections on the benign gynecology service. Women with genital malignancies are at high risk for the development of a variety of infections by resistant pathogens, emphasizing the importance of obtaining cultures prior to initiation of therapy and carefully selecting the antibiotics to be prescribed. 1990 Academic Press, Inc.
\end{abstract}

Genital malignancy constitutes approximately onefourth of invasive cancers diagnosed in women. Therapy oftentimes consists of a combination of surgery, radiation therapy, and chemotherapy. These patients may be at high risk for infectious morbidity for a number of reasons. Frequently patients with endometrial cancer are obese and, at a referral center such as the University of Michigan Medical Center, weigh more than $\mathbf{3 0 0}$ pounds. The increased use of lymph node dissection in surgery for pelvic malignancies extends the time of operation and allows for accumulation of blood and body fluid in devascularized tissue. Patients with ovarian carcinoma and those undergoing pelvic exenteration present the possibility for a unique bacteriologic spectrum to be encountered: not only will the vagina be entered, but when bowel surgery is required the patient is at risk for bacterial contamination from multiple sites. These patients

\footnotetext{
${ }^{1}$ To whom reprint requests should be addressed.
}

are usually in an older age group and may have a depressed immune status. Lastly, the nutritional status of these patients is often borderline, especially in the obese patient or in women with advanced stages of cancer.

During the past two decades, great advances have been made in understanding the microbiology, pathophysiology, and treatment of the common obstetric and gynecologic infections, including postpartum endoparametritis, pelvic cellulitis, and other community acquired infections such as pelvic inflammatory disease. It must be assumed that the majority of clinicians have relied on these data when treating the gynecologic oncology patient. With the use of radiation therapy, chemotherapy, and extended surgery in these patients, it seems unwise to make this assumption. Since the report by Ledger $e t$ al. [1] of infectious morbiidty and sepsis on a gynecology service in the early 1970s, little additional information has been made available with respect to gynecologic oncology patients. Thus, a yearlong survey of bacterial infections on a gynecologic oncology service was undertaken; additionally, bacterial isolates from the oncology patients are compared to pathogens isolated from women developing infections hospitalized on the benign gynecology service during the same period.

\section{MATERIALS AND METHODS}

From January 1, 1986, thru December 31, 1986, all patients admitted to the gynecologic oncology service at the University of Michigan Medical Center were prospectively monitored for the development of infections acquired during hospitalization. Patients were listed in a registry on the day of admission and charts were reviewed on a daily basis. Following discharge, the charts were again reviewed and microbiologic data gathered. The diagnosis of infection was based on clinical and laboratory parameters. Cultures including blood, urine, wound, abscess, operative site, intravenous lines, and 
miscellaneous body fluids were obtained in nonsurgical patients exhibiting temperature greater than $38^{\circ} \mathrm{C}$ and in postoperative patients with temperature greater than $38^{\circ}$ $\mathrm{C}$ on two occasions $6 \mathrm{hr}$ apart excluding the first $24 \mathrm{hr}$. Infectious sites were defined by using the following criteria:

\section{Urinary tract}

Wound

Pneumonia

Pelvic cellulitis

All patients with a bacterial growth of greater than $10^{5}$ colonies of a single pathogen per milliliter of urine in midstream clean-voided specimen. Urine specimens obtained by urinary catheter yielding one or more uropathogens were felt to represent bacteriuria.

All inflamed surgical wounds with purulent drainage and confirmed by microbiologic studies.

Patients with physical examination and $\mathrm{x}$-ray findings consistent with pneumonia, most were confirmed by gram stain and culture.

Postoperative patients exhibiting pyrexia, lower abdominal pain, tenderness and induration on pelvic exam with supporting laboratory studies (including leukocytosis). A Specimen for culture was not routinely obtained from the vaginal cuff on the oncology or benign gynecology service.

Microbiology. Microorganisms were isolated and identified by standard microbiologic techniques. Sensitivities were determined by a microdilution method.

Antimicrobial prophylaxis. Parenteral prophylactic antibiotics included cefazolin $1 \mathrm{~g}$ every $8 \mathrm{hr}$ or cefoxitin 1-2 $\mathrm{g}$ every $6 \mathrm{hr}$ for $24-48 \mathrm{hr}$ at the discretion of the attending physician. Patients with significant risk for bowel entry routinely had bowel cleansing consisting of erythromycin $500 \mathrm{mg}$ and neomycin $1 \mathrm{~g}$ orally every 6 $\mathrm{hr}$ for at least $24 \mathrm{hr}$ prior to surgery and a neomycin enema the morning of surgery.

Statistical Analysis. Data were analyzed using the $\chi^{2}$ or Fishers's exact test where appropriate.

\section{RESULTS}

During the study period there were a total of 510 admissions to the gynecologic oncology service involving 297 patients. The most common reason for admission was surgery $(n=218)$, followed by chemotherapy ( $n=$ $192)$ and radiation therapy $(n=38)$. Patient admissions by origin of malignancy are noted in Table 1 .

There were 109 bacteriologically confirmed infections in the study group (Table 2). An additional 31 patients
TABLE 1

Patients Admitted by Origin of Malignancy $(N=297)$

\begin{tabular}{lr}
\hline Cervix & 99 \\
Ovary & 98 \\
Uterus & 48 \\
Vulva & 28 \\
Fallopian tube & 8 \\
Vagina & 4 \\
Uncertain & 12 \\
\hline
\end{tabular}

received empiric therapy for pelvic cellulitis or urinary tract infection not confirmed by microbiologic studies. Urinary tract infection $(n=54)$ was the most common microbiologically confirmed infection followed by wound infection $(n=22)$ and pneumonia $(n=15)$. Six of eight patients with multiple infections $(n=20)$ had undergone radical pelvic procedures, including total pelvic exenteration $(n=3)$, radical vulvectomy $(n=2)$, and radical hysterectomy $(n=1)$.

There were 136 organisms isolated from the patients (Table 3). Escherichia coli was the most common of 26 different isolates. Other frequently isolated organisms include Pseudomonas aeruginosa and group D enterococcus.

During the study period 525 patients were admitted to the benign gynecology service. The bacteria isolated from these women developing infections were significantly different in the frequency of recovery (Table 4) and antibiotic sensitivity trends (Table 5) from those in oncology patients. $E$. coli was the most common organism isolated from both patient groups and was more frequently recovered from benign gynecology patients $(P$ $<0.0005)$. $E$. coli isolates from both services were similar with respect to sensitivity to commonly prescribed antimicrobials including ampicillin, cefoxitin, and gentamicin. $P$. aeruginosa represented $15 \%$ of total isolates from the oncology infections although there were only $3(3.2 \%)$ from infections in benign gynecology patients $(P<.01)$. Isolates from both services were highly resistant to first- and second-generation cephalosporins, but were sensitive to most third-genertion cephalosporins. All isolates from benign gynecology patients were sensitive to gentamicin; however, $25 \%$ of $P$. aeruginosa

TABLE 2

Bacteriologically Confirmed Infections in Gynecologic Oncology Patients $(N=109)$

$\begin{array}{lr}\text { Urinary tract infection } & 54 \\ \text { Wound Infection/Cellulitis } & 26 \\ \text { Pneumonia } & 15 \\ \text { Bacteremia } & 8 \\ \text { Abscess } & 4 \\ \text { Peritonitis } & 1 \\ \text { Colitis } & 1\end{array}$


TABLE 3

Pathogens Isolated from Gynecologic Oncology Patients (Total and Source)

\begin{tabular}{lrrcc}
\hline Organism & Total & Urine & Wound & Other \\
\hline Escherichia coli & 26 & 18 & 5 & 3 \\
Pseudomonas aeruginosa & 18 & 9 & 6 & 3 \\
Group D enterococcus & 16 & 8 & 6 & 2 \\
Staphylococcus epidermidis & 15 & 10 & & 5 \\
Klebsiella pneumoniae & 8 & 3 & 1 & 4 \\
Enterobacter cloacae & 7 & 2 & 3 & 2 \\
Candida albicans & 7 & 6 & & 1 \\
Proteus vulgarts & 5 & 5 & & \\
Proteus mirabilis & 7 & 4 & 3 & \\
Staphylococcus aureus & 5 & 2 & 2 & 1 \\
Clostridium perfringens & 3 & & & 3 \\
Klebsiella oxytoca & 2 & & & 2 \\
Bacteroides fragilis & 2 & & 2 & \\
Citrobacter diversus & 2 & & & \\
Enterobacter aerogenes & 1 & & 1 & \\
Morganella morgani & 1 & 1 & & \\
Providencia stuartii & 1 & 1 & & \\
Pseudomonas putida & 1 & 1 & & \\
Lactobacillus sp. & 1 & 1 & & \\
Diptheroids & 1 & 1 & & 1 \\
Capnocytophagia sp. & 1 & & & 1 \\
Penicillium sp. & 1 & & & 1 \\
Haemophilus influenzae & 1 & & & 1 \\
Clostridium ramosum & 1 & & & 1 \\
Peptostreptococcus sp. & 1 & & & 1 \\
Acinetobacter sp. & 1 & & & 1 \\
Group B streptococccus & 1 & & & \\
\hline
\end{tabular}

isolates from oncology patients were resistant to gentamicin. Additionally, $36 \%$ of gentamicin-sensitive $P$. aeruginosa isolates were determined to be of intermediate sensitivity to gentamicin as determined by microdiluation method. Of other facultative gram-negative rods, Proteus sp. and Klebsiella sp. were sensitive to first- and second-generation cephalosporins. Enterobacter cloacae were resistant to first- and second-generation cephalosporins and one $E$. cloacae isolate was resistant to several third-generation cephalosporins tested. Both

TABLE 4

Pathogens Isolated from Benign Gynecology Patients

\begin{tabular}{lrrrr}
\hline Organism & Total & Urine & Wound & Other \\
\hline Escherichia coli & 42 & 29 & 4 & \\
Proteus mirabilis & 6 & 5 & & \\
Citrobacter freundi & 4 & 3 & & \\
Klebsiella pneumoniae & 9 & 3 & 1 & \\
Pseudomonas aeruginosa & 3 & 2 & & \\
Enterobacter cloacae & 3 & 2 & & \\
Entcrococcus & 8 & & 2 & \\
Coagulase-positive staphylococcus & 4 & 2 & 1 & 1 \\
Coagulase-negative staphylococcus & 11 & 6 & 2 & 2 \\
Bacteroides fragilis & 8 & 3 & 1 & \\
Other & & &
\end{tabular}

TABLE 5

Comparison of Sensitivity Patterns of Pathogens Isolated for Patients on the Gynecology Services

\begin{tabular}{|c|c|c|c|}
\hline \multirow[b]{2}{*}{ Isolate } & \multirow[b]{2}{*}{ Antibiotic } & \multicolumn{2}{|c|}{$\begin{array}{c}\text { Percentage of } \\
\text { isolates sensitive on } \\
\text { respective } \\
\text { gynecology service }\end{array}$} \\
\hline & & Benign & Oncology \\
\hline \multirow{2}{*}{$\begin{array}{l}\text { Coagulase-positive } \\
\text { staphylococci }\end{array}$} & Penicillin & 0 & 0 \\
\hline & Vancomycin & 100 & 100 \\
\hline \multirow{2}{*}{$\begin{array}{l}\text { Coagulasc-ncgative } \\
\text { staphylococci }\end{array}$} & Penicillin & 20 & 0 \\
\hline & Vancomycin & 100 & 100 \\
\hline \multirow{3}{*}{ Escherichia coli } & Ampicillin & 67 & 75 \\
\hline & Cefoxitin & 90 & 100 \\
\hline & Gentamicin & 100 & 100 \\
\hline \multirow[t]{2}{*}{ Pseudomonas aeruginosa } & Cefoxitin & 0 & 0 \\
\hline & Gentamicin & 100 & 75 \\
\hline
\end{tabular}

coagulase-positive and coagulase-negative staphylococci were frequently isolated from both services, with isolates from the benign gynecology service exhibiting modest sensitivity to commonly prescribed penicilins and cephalosporins. Isolates from oncology patients were uniformly resistant to all antimicrobials except vancomycin.

\section{DISCUSSION}

Since the report by Ledger et al. [1] in 1972, little has been reported on infections in gynecologic oncology patients. In the ensuing 17 years, however, significant advances in the care of women with genital malignancies have occurred, including surgical technique and selection of surgical candidates, introduction of new chemotherapeutic agents, introduction of new broad-spectrum antibiotics, and the routine use of perioperative prophylactic antibiotics. Ledger et al. studied the infectious morbidity on the Gynecology Service at the University of Michigan Medical Center that included 119 patients with genital malignancies; nearly half $(n=50)$ were admitted for medical indications or radiation therapy. In their report, $52 \%$ of women with genital malignancies admitted for nonsurgical reasons experienced febrile morbidity and $36 \%$ received antibiotics. In the current report, infections were diagnosed in $5.7 \%$ of admissions for chemotherapy and $13 \%$ of admissions for radiation therapy. Reasons for this reduction in infectious morbidity are unclear; however, they may include improvements in infection control and more experience with chemotherapeutic agents. Since we studied all patients prospectively, underreporting of infections in the current series is not possible.

Ledget et al. noted the prevalence of postoperative infections to be highest among oncology patients. Of 69 surgical patients in his series, $56(81 \%)$ experienced fe- 
brile morbidity while $54(78 \%)$ received antibiotics. $\mathrm{He}$ noted patients undergoing radical surgical procedures to be at highest risk for infectious morbidity $(86 \%)$. Of the 218 admissions for surgery in the current study, $75 \mathrm{mi}-$ crobiologically confirmed infections occurred in $62(28 \%)$ patients, while $31(14 \%)$ patients received empiric therapy for suspected pelvic cellulitis or urinary tract infection. After allowing for multiple infections, the infectious morbidity in surgical patients is approximately $43 \%$, half that reported by Ledger et al. In a retrospective study of hospital admissions of gynecologic oncology patients, Brooker et al. [2] reported that $11 \%$ of patients developed serious infections, which was not defined. Due to the retrospective nature of this study and the exclusion of uncomplicated urinary tract infections and other "less serious" infections from the data base, the overall infectious morbidity would be underestimated. The current prospective study included all microbiologically confirmed infections and presumed postoperative infections such as pevic cellulitis and may be more representative of infectious morbidity in women with gential malignancy.

Procedures with the potential for lower morbidity, i.e., staging laparotomy for cervical cancer and second-look laparotomy for ovarian carcinoma, may contribute to the lower infection rate in the current study. Other retrospective studies report a low incidence of infectious morbidity in paticnts undergoing radical hystcrectomy [3] (14\% major and minor infections), although infections were common in patients undergoing pelvic exenteration (pelvic cellulitis $70 \%$, abdominal wound infection $28 \%$, pyelonephritis $15 \%$, and sepsis $15 \%$ ) [4].

A likely factor in the reduction of infectious morbidity during this period is the use of perioperative antimicrobial prophylaxis, administered prior to 181 of $218(83 \%)$ procedures. Investigators have demonstrated the benefit of a bowel prep as used in this series in reducing morbidity in bowel surgery [5]. Parenteral prophylactic antibiotics have not been studied extensively in radical pelvic surgery. There are no prospective controlled trials evaluating the efficacy of parenteral prophylactic antibiotics for radical vulvectomy or pelvic exenteration, although these procedures appear to be associated with a very high risk for infectious morbidity to justify parenteral antimicrobials. In placebo controlled studies, Rosenshein et al. [6] found single-dose vibramycin to significantly reduce the incidence of febrile morbidity and infectious morbidity in patients undergoing radical hysterectomy; likewise, Micha et al. [7] found three-dose mezlocillin to significantly reduce the fever index and the incidence of operative site infections. Certainly additional studies are necessary to further define the role and duration of administration of prophylactic antibiotics in this population at high risk for infection.
Traditionally, obstetric and gynecologic infections are considered mixed polymicrobial infections. Frequently isolated organisms include anaerobic gram-positive cocci, anaerobic gram-negative rods, facultative gramnegative rods including $E$. coli, and group $\mathrm{B} \beta$-hemolytic streptococcus. In a study in obstetric and benign gynecologic patients, Herd et al. reported that 204 of 208 gram-negative rods were sensitive to gentamicin at $\leqslant 4$ $\mu \mathrm{g} / \mathrm{ml}[8]$. As in our report, $E$. coli was the most frequent gram-negative isolate and except for ampicillin, antibiotic resistance was uncommon. Of note, approximately onefifth of the gram-negative rods recovered from cancer patients and tested for susceptibility to gentamicin had minimum inhibitory concentrations exceeding $4 \mu \mathrm{g} / \mathrm{ml}$ $(P<0.025)$. Resistant $P$. aeruginosa is an uncommon pathogen in healthy patients and seems unique to this population. Enterococcus was isolated frequently from women on both gynecology services $(P>0.05)$ and likely reflects the widespread use of cephalosporin prophylaxis as was recently described by Faro et al. [9] Factors contributing to the high prevalence of these organisms or antibiotic resistance in gynecologic oncology patients include (1) the need for multiple and prolonged hospitalizations allowing for colonization, (2) prior antibiotic therapy or prophylaxis allowing for induction of antimicrobial resistance or predisposing to overgrowth or superinfection with hospital-acquired pathogens, and (3) underlying malignancy or chemotherapy altering host resistance. It is most important to recognize that infections in gynecologic oncology patients are frequently caused by resistant pathogens and proper selection of antimicrobial agents should reflect this awareness. After empiric therapy is initiated, it is imperative that results of cultures be obtained, sensitivities determined, and the most appropriate antibiotic prescribed.

In summary, this study defines the infectious morbidity on a gynecologic oncology service. It appears that over a 17-year period, the prevalence of infectious morbidity has declined at the authors' institution. Many of the infections and the microbiolgoy are unique to this patient population. Additional studies are needed to further define infectious morbidity as well as antimicrobial therapy and prophylaxis in women with genital malignancy.

\section{REFERENCES}

1. Ledger, W. J., Reite, A., and Headington, J. T. Infection on an inpatient gynecology service, Amer.J. Obstet. Gynecol. 113(5), 662671 (1972).

2. Brooker, D. C., Savage, J. E., Twiggs, L. B., Adcock, L. L., Prem, K. A., and Sanders, C. C. Infectious morbidity in gynecologic cancer, Amer. J. Obstet. Gynecol. 156, 513 (1987).

3. Lerner, H. M., Jones, H. W., and Hill, E. C. Radical surgery for early invasive cervical carcinoma (stage IB): Review of 15 years experience, Obstet. Gynecol. 56, 413 (1980). 
4. Morgan, L. S., Daly, J. W., and Monif, G. R. G. Infectious morbidity associated with pelvic exenteration, Gynecol. Oncol. 10, 318 (1980).

5. Clarke, J. S., Condon, R. E., Bartlet, J. G., Gorbach, S. L., and Nichols, R. L. Preoperative oral antibiotics reduce septic complications of colon operations: Results of prospective, randomized, double-blind clinical study, Ann. Surg. 186, 251-259 (1979).

6. Rosenshein, N. B., Ruth, J. C., Villar, J., Grumbine, F. B., Dillon, M. B., and Spence, M. R. A prospective randomized study of doxycycline as a prophylactic antibiotic in patients undergoing radical hysterectomy, Gynecol. Oncol. 15, 201 (1983).
7. Micha, J. P., Kucera, P. R., Birkett, J. P., Chambers, G., Sheets, E. E., and DiSaia, P. J. Prophylactic mezlocillin in radical hysterectomy, Obstet. Gynecol. 69, 251 (1987).

8. Herd, M. L., Bowden, R. E., and Hemsell, D. Susceptibility profiles of potential of aerobic and anaerobic isolates for hysterectomy patients, Amer. J. Obstet. Gynecol., 149, 133-143 (1984).

9. Faro, S., Martens, M., Hammill, H., Phillips, E. L., Smith, D., and Riddle, G. Ticarcillin/clavulanic acid versus clindamycin and gentamicin in the treatment of post-cesarean endometriosis following antibiotic prophylaxis, Obstet. Gynecol. 73, 808-812 (1989). 